\title{
Medicinal Mushroom Science: Current Perspectives, Advances, Evidences, and Challenges
}

\author{
Solomon P. Wasser ${ }^{1,2}$
}

\begin{abstract}
The main target of the present review is to draw attention to the current perspectives, advances, evidences, challenges, and future development of medicinal mushroom science in the $21^{\text {st }}$ century. Medicinal mushrooms and fungi are thought to possess approximately 130 medicinal functions, including antitumor, immunomodulating, antioxidant, radical scavenging, cardiovascular, anti-hypercholesterolemic, antiviral, antibacterial, anti-parasitic, antifungal, detoxification, hepatoprotective, and antidiabetic effects. Many, if not all, higher Basidiomycetes mushrooms contain biologically active compounds in fruit bodies, cultured mycelium, and cultured broth. Special attention is paid to mushroom polysaccharides. The data on mushroom polysaccharides and different secondary metabolites are summarized for approximately 700 species of higher hetero- and

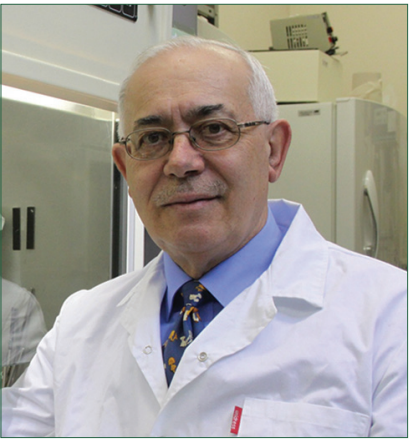

Prof. Solomon P. Wasser homobasidiomycetes. Numerous bioactive polysaccharides or polysaccharide-protein complexes from the medicinal mushrooms described appear to enhance innate and cell-mediated immune responses, and exhibit antitumor activities in animals and humans. Whilst the mechanism of their antitumor actions is still not completely understood, stimulation and modulation of key host immune responses by these mushroom compounds appear central. Polysaccharides and low-molecular-weight secondary metabolites are particularly important due to their antitumor and immunostimulating properties. Several of the mushroom compounds have been subjected to Phase I, II, and III clinical trials, and are used extensively and successfully in Asia to treat various cancers and other diseases. Special attention is given to many important unsolved problems in the study of medicinal mushrooms.
\end{abstract} (Biomed J 2014;37:345-356)

\section{Key words: antioxidant activities, antitumor, $\beta$-glucans, immunomodulating, medicinal mushrooms, polysaccharides, secondary metabolites}

$\mathrm{T}^{\mathrm{s}}$ he use of mushrooms in traditional ancient therapies dates back at least to the Neolithic age. For millennia, mushrooms have been valued as edible and medical provisions for humankind. Contemporary research has validated and documented much of the ancient knowledge on medicinal mushrooms (MMs). An interdisciplinary field of science that focuses on MMs has been developed and increasingly demonstrates potent and unique properties of compounds extracted from a range of mushroom species in the last three decades. Modern clinical practice in Japan, China, Korea, Russia, and several other countries rely on mushroom-derived preparations. ${ }^{[1-4]}$
Ancient oriental traditions have stressed the importance of several mushroom species, namely, Ling Zhi or Reishi [Ganoderma lucidum (W.Curt.:Fr.) P. Karst.] and Shiitake mushrooms [Lentinus edodes (Berk.) Singer]. Mushrooms have also played an important role in the treatment of ailments affecting the rural populations of eastern European countries. The most important species in these countries are Inonotus obliquus (Pers.:Fr.) Pilát (Chaga), Fomitopsis officinalis (Vill.:Fr.) Bond. et Singer (wood conk or agaricon), Piptoporus betulinus (Bull.:Fr.) P. Karst. (birch polypore), and Fomes fomentarius Fr.:Fr (tinder bracket). ${ }^{[4,5]}$ These species are used in the treatment of gastrointestinal

From the ${ }^{1}$ Institute of Evolution and Department of Evolutionary and Environmental Biology, Faculty of Natural Sciences, University of Haifa, Israel; ${ }^{2}$ Department of Cryptogamic Plants and Fungi, N. G. Kholodny Institute of Botany, National Academy of Sciences of Ukraine, Ukraine Received: Apr. 30, 2014; Accepted: Jul. 17, 2014

Correspondence to: Dr. Solomon P. Wasser, Institute of Evolution and Department of Evolutionary and Environmental Biology, Faculty of Natural Sciences, University of Haifa, Israel. 199 Abba Khousi Ave., Mount Carmel, Haifa 3498838, Israel. Tel: 972-4-8249218; Fax: 972-4-8288649; E-mail: spwasser@ research.haifa.ac.il

DOI: $10.4103 / 2319-4170.138318$ 
disorders, various forms of cancers, bronchial asthma, night sweats, etc., There is also a long history of traditional use of mushrooms as curatives in Mesoamerica (especially for species of the genus Psilocybe), Africa (Yoruba populations in Nigeria and Benin), Algeria, and Egypt. A very special role of fly agaric [Amanita muscaria (L.:Fr.) Pers.] is found in Siberia and Tibetan shamanism, Buddhism, and Celtic myths. ${ }^{[1,2,4,6]}$

Nowadays, MMs are used as: (A) Dietary food (world mushroom production was 30 million tons in 2012); (B) dietary supplement (DS) products (the market of MM DS products is quickly growing and has a value of more than 18 billion US dollars per year); (C) a new class of drugs called "mushroom pharmaceuticals;" (D) natural bio-control agents in plant protection with insecticidal, fungicidal, bactericidal, herbicidal, nematicidal, and antiphytoviral activities; and (E) cosmeceuticals, different compounds of MMs, including polysaccharides such as soluble $\beta$-glucans, glucuronoxylomannan (GXM), sacchachitin, tyrosinase and other enzymes, which are used by cosmetic companies for their film-forming capability, activation of epidermal growth factor, antioxidative, anti-allergic, antibacterial, and anti-inflammatory activities, stimulation of collagen activity, inhibition of autoimmune vitiligo, and treatment of acne. ${ }^{[3,4,7]}$

MMs are comparable to "medicinal plants" and can be defined as macroscopic fungi, mostly higher Basidiomycetes and some Ascomycetes, which are used in the form of extracts or powder for prevention, alleviation, or healing of diseases, and/or in providing a balanced healthy diet. According to the definition of "herbal drugs," dried fruit bodies, mycelia, or spores are considered "mushroom drugs" or "fungal drugs." Analogous to "phytopharmaceuticals" or "herbal preparations," the resulting preparations should be considered as "mushroom pharmaceuticals" or "mushroom preparations." "[8]

Fungi and mushrooms are extremely abundant worldwide and show diversity. Recent estimates of the number of fungi on earth show it to range from 500,000 to more than 5 million species, and the generally accepted estimate was 1.5 million, according to a report published more than 20 years ago. ${ }^{[9]}$ To date, it is recommended that as many as 3 million species of fungi be accepted for general use. ${ }^{[10]}$ Meanwhile, currently 110,000 species of fungi of all kinds have been described. The figure is based on the total obtained by adding the number of species in each genus given in the last edition of the Dictionary of Fungi ${ }^{[11]}$ published in previous years, ${ }^{[10,12,13]}$ and includes all organisms traditionally studied by mycologists: Slime molds, chromistan fungi, chytridiaceous fungi, lichen-forming fungi, filamentous fungi, molds, and yeasts. Out of these, mushrooms constitute 16,000 species, calculated from the Dictionary of Fungi published in previous years. ${ }^{[1]]}$ The number of mushroom species on earth is currently estimated at $150,000-160,000$; yet, perhaps only $10 \%$ of the named species are known to science. ${ }^{[3,9-10,11]} \mathrm{An}$ analysis of the localities from which fungi new to science have been described and cataloged in the Index of Fungi in the last 10 years revealed that about $60 \%$ of all newly described fungi are from the tropics. This is also the case for mushrooms, especially those species forming ectomycorrhizas with native trees, although new species continue to be discovered in Europe and North America. In various tropical areas, $22-55 \%$ (in some cases, up to $73 \%$ ) of mushroom species have not yet been described..$^{[9,10,13]}$ The approximate real number of the species of fungi existing on earth can be 50 times higher than the data, based on recent calculations. Modern sequencing methods suggest that as many as 5 million fungal species exist. ${ }^{[12]}$ Therefore, we would need more than 4000 years to describe this fungal diversity based on the present discovery rate of about 1200 new species per year in the last 10 years of data. ${ }^{[14]}$ The data also show that we are familiar with approximately $1 \%$ of the world fungal biota and only $10 \%$ of the world's mushroom biodiversity. ${ }^{[3,4]}$

Specialists in taxonomy of some groups of MMs are familiar with "known" species, but some of their biochemical and pharmacological properties remain hidden. The author ${ }^{[3]}$ has summarized the data on approximately 700 mushroom species, with the pharmacological properties of 2000 known, safe species. It again shows that the present state of knowledge presents the great potential of MM diversity.

Mushrooms are currently evaluated for their nutritional value and acceptability, as well as for their pharmacological properties. They make up a vast and yet largely untapped source of new powerful pharmaceutical products. In particular, and most importantly for modern medicine, MMs present an unlimited source of polysaccharides (especially $\beta$-glucans) and polysaccharide-protein complexes with anticancer and immunostimulating properties. Many, if not all, higher Basidiomycetes mushrooms contain many types of biologically active high-molecular-weight and low-molecular-weight compounds (triterpenes, lactones, alkaloids, and other compounds) in fruit bodies, cultured mycelia, and cultured broth. ${ }^{[3,4,15-17]}$

\section{Current perspectives and advances}

MMs and fungi are thought to possess approximately 130 medicinal functions. Recently studied medicinal actions of mushrooms include antitumor, immunomodulating, antioxidant, radical scavenging, cardiovascular, antihypercholesterolemic, antiviral, antibacterial, anti-parasitic, antifungal, detoxicating, hepatoprotective, and antidiabetic effects. The best usage of MM drugs and MM DSs has been in preventing and treating immune disorders, especially in immunodeficient and immunosuppressed patients; they are also used for patients under 
chemotherapy or radiotherapy; in different types of cancers, chronic blood-borne viral infections of Hepatitis B, C, and $\mathrm{D}$, different types of anemia, the human immunodeficiency virus/acquired immunodeficiency syndrome (HIV/AIDS), Herpes simplex virus (HSV), chronic fatigue syndrome, Epstein-Barr virus; for patients with chronic gastritis and gastric ulcers caused by Helicobacter pylori; and for patients with dementia (especially for Alzheimer's disease). ${ }^{[3,4,18-20]}$

Mushroom polysaccharides prevent oncogenesis, show direct antitumor activity against various synergetic tumors, and prevent tumor metastasis. Their activity is especially beneficial when used in conjunction with chemotherapy. The antitumor action of polysaccharides requires an intact T-cell component; their activity is mediated through a thymus-dependent immune mechanism. They activate cytotoxic macrophages, monocytes, neutrophils, natural killer (NK) cells, dendritic cells, and chemical messengers (cytokines such as interleukins, interferons, and colony stimulating factors) that trigger complementary and acute phase responses. Also, mushroom polysaccharides can be considered as multi-cytokine inducers able to induce gene expression of various immunomodulating cytokines and cytokine receptors. ${ }^{[3,21-25]}$

Cancer has probably always existed in mankind; it is probably as old as life. Evidence of cancer was reported in a Neanderthal skull (35,000 BC) and in Egyptian and Incan mummies. ${ }^{[26]}$ Cancer is a broad term that includes hundreds of different types of diseases that can develop in the body. It is a generic term used for malignant neoplasms.

The new figures and projections of the global cancer burden presented in the new edition of the World Cancer Report $^{[27]}$ starkly highlight the problem: The incidence of cancer has increased from 12.7 million in 2008 to 14.1 million in 2012, and this trend is projected to continue, with the number of new cases expected to rise in the future to $75 \%$. Among men, the five most common sites diagnosed with cancer in 2012 were the lung (16.7\% of the total), prostate $(15.0 \%)$, colorectum $(10 \%)$, stomach $(8.5 \%)$, and liver $(7.5 \%)$; lung cancer had the highest incidence $(34.2$ per 100,000$)$ and prostate cancer had the second highest incidence $(31.1$ per 100,000). Among women, the five most common sites affected by cancer were the breast (25.2\% of the total), colorectum (9.2\%), lung (8.7\%), cervix $(7.9 \%)$, and stomach $(4.8 \%)$; breast cancer had a substantially higher incidence $(43.3$ per 100,000$)$ than any other cancer and the next highest incidence was of colorectal cancer (14.3 per 100,000). Among the major non-communicable diseases (the four major diseases - cardiovascular disease, chronic diabetes, respiratory diseases, and cancer), at the national, regional, and global levels, cancer has been the main cause of death around the world in the last few years. ${ }^{[27]}$
A total of more than 40 million new cancer cases are predicted in the world for this year. The estimates reported by WHO indicate that 84 million people will die of cancer between 2005 and 2015. Cancer is killing more people than AIDS, malaria, and tuberculosis together. Additionally, in China and India (the most populated countries), cancer deaths are increasing, largely because of smoking, diet, and ecology problems. Globally, the number of deaths from cancer, according to WHO, is expected to reach 17 million/ per year in 2030. ${ }^{[27]}$

$\mathrm{MM}$ drugs and MM polysaccharide preparations from different mushroom species show positive results in treating cancers in vitro and in vivo. A new class of antitumor MM drugs is called biological response modifiers (BRMs). BRMs have been used as a new kind of cancer treatment, along with surgery, chemotherapy, and radiotherapy. ${ }^{[3,4,23-25,28]}$ The major problem caused by cancer treatments, especially chemotherapy and radiotherapy, is damaging or weakening of the patient's natural immunological defenses. MM BRMs treat cancers, focusing on improving the patient's quality of life, since they significantly reduce the side effects and help overcome cancer growth. Most of them activate natural immune responses of the host and can be used as supportive treatment for cancer prevention and, in some cases, alone with conventional therapies.

Immunoceuticals isolated from more than $30 \mathrm{MM}$ species have demonstrated antitumor activity in animal treatments. However, only a few have been tested for their anticancer potential in humans. The few that have been tested are $\beta$-d-glucans or $\beta$-d-glucans linked to proteins. Moreover, the latter have shown greater immunopotentiation activity than the free glucans. There are several clinical studies proving the cancer inhibitory effects of L. edodes, ${ }^{[21,23,29]}$ Grifola frondosa (Dicks.:Fr.) Gray, ${ }^{[30,31]}$ Schizophyllum commune Fr.:Fr., ${ }^{\left[24,{ }^{32]}\right.}$ Ga. lucidum ${ }^{[33,34]}$ Trametes versicolor (L.:Fr.) Lloyd, ${ }^{[35]}$ I. obliquus, ${ }^{[36,37]}$ Phellinus linteus (Berk. et M.A. Curt.) Teng, ${ }^{[38]}$ Flammulina velutipes (W.Curt.:Fr.) Singer, ${ }^{[39]}$ Hypsizygus marmoreus (Peck) Bigel., ${ }^{[40]}$ Ophicordyceps (=Cordyceps) sinensis (Berk.) G. H. Sung et al. ${ }^{[41]}$ Agaricus brasiliensis S. Wasser et al. (=Agaricus blazei sensu Heinem.), ${ }^{[42,43]}$ and Tremella mesenterica Retz.:Fr. ${ }^{[19,44]}$ Mushroom immunoceuticals act mainly by improving the host immune system. This process includes activation of dendritic cells, NK cells, T-cells, macrophages, and production of cytokines. Several MM products, mainly polysaccharides (especially $\beta$-glucans), were developed for clinical and commercial purposes: Krestin (PSK) and PSP (polysaccharide peptide) from T. versicolor; Lentinan, isolated from L. edodes; Schizopyllan (Sonifilan, Sizofiran, or SPG) from S. commune; Befungin from I. obliquus; D-fraction, from Gr. frondosa; GLPS polysaccharide fraction from Ga. lucidum; active hexose correlated compound (AHCC); and many others. 
Other mushroom compounds of therapeutic interest are the secondary metabolites, especially some low-molecular-weight compounds such as lactones, terpenoids, and alkaloids, antibiotics with different chemical groups, and metal chelating agents, which are also important for the immune function of the organism. MMs also contain a number of enzymes such as laccase, superoxide dismutase, glucose oxidase, and peroxidase. It has been shown that enzyme therapy plays an important role in cancer treatment by preventing oxidative stress and inhibiting cell growth. ${ }^{[3,16]}$

It has been documented that fungi produce a huge number of biologically active compounds that not only stimulate the immune system but also modulate specific cellular responses by interfering in particular transduction pathways. For instance, the caffeic acid phenethyl ester (CAPE), which specifically inhibits the DNA binding of nuclear factor kappa-light-chain-enhancer of activated $\mathrm{B}$ cells $(\mathrm{NF}-\mathrm{KB})$ and has shown some promising results in human breast cancer MCF-7 cells, was found to be produced by Agaricus bisporus (J.Lge) Imbach, Marasmius oreades (Bolt.) Fr., L. edodes, and Ph. linteus. Also, a methanol extract of $F$. fomentarius was reported to inhibit inducible nitric oxide synthase (iNOS) and cyclooxygenase (COX) expression due to the downregulation of NF- $\mathrm{KB}$ binding activity to DNA. Panepoxydone, a compound isolated from Panus spp. but also found in Lentinus crinitus, interferes with the NF- $\kappa \mathrm{B}$-mediated signal by inhibiting the phosphorylation of IкB $\alpha$ (Inhibitory protein kappa $\alpha$ ). These reports demonstrate the fact that such substances can be used as molecular targets in malignant cells in order to combat cancer. Because low molecular sizes help them to penetrate the cell membrane, these substances have also been classified as low-molecular-weight compounds; among them are lectins, lactones, terpenoids, alkaloids, antibiotics, and metal chelating agents. Many fungal species have already been reported to produce various metabolites capable of modulating different intracellular pathways, thus playing an essential role in cancer treatment. ${ }^{[3,4,16,45-50]}$

MMs produce beneficial effects not only as drugs but also as a novel class of products with different names: Dietary supplements, functional foods, nutriceuticals, mycopharmaceuticals, and designer foods including probiotics and prebiotics, which provide healthy benefits through everyday use.

DSs are not strictly used as pharmaceutical products; they also produce healthy effects through everyday use as part of a healthy diet.

Several types of MM DS products are available on the market today.

1. Artificially cultivated fruit body powders, hot water or alcohol extracts of these fruit bodies

2. Dried and pulverized preparations of the combined substrate, mycelium, and mushroom primordial
3. Biomass or extracts from mycelium harvested from submerged liquid culture grown in a fermentation tank or bioreactor

4. Naturally grown, dried mushroom fruit bodies in the form of capsules or tablets

5. Spores and their extracts..$^{[4,8]}$

The increased interest in traditional remedies for various physiological disorders and the recognition of numerous biological activities of mushroom products have led to the coining of the term "mushroom nutriceuticals," which should not be confused with nutraceuticals, functional foods, and pharmaceuticals. A mushroom nutriceutical is a refined, or partially refined, extract or dried biomass from either mycelium or the fruiting body of a mushroom, which is consumed in the form of capsules or tablets as a DS (not a food) and has potential therapeutic applications. Regular intake may enhance the immune response of the human body, thereby increasing resistance to disease and, in some cases, causing regression of the disease state. Thus, acting as immunopotentiators, MM preparations modify host biological responses (also known as BRMs).

There is no doubt that MM-based products can serve as superior DSs. The market of DS from mushrooms is quickly growing and is valued at more than 18 billion US dollars (representing 10\% of the general market of DSs). Every year, data are collected as new evidence on the beneficial effects from DSs made from MMs ${ }^{[4]}$ A new product for dementia (especially for Alzheimer's disease) based on a proprietary standardized extract that contains hericenones and amyloban (both from the Hericium erinaceus - lion's mane mushroom) is currently available on the market. The value of Ganoderma, Ophiocordyceps, and Cordyceps DSs alone is more than 4.0 billion USD per year.

\section{Evidences, challenges, and unsolved problems}

On the one hand, MM science made great progress in the last 30 years. A really successful new branch of science (Medicinal Mushroom Science) has been recognized.

New classes of MM drugs and different types of MM products have been developed. A unique journal in the field, the International Journal of Medicinal Mushrooms (Begell House, USA) was organized. Every 2 years, an International Medicinal Mushroom Conference is organized; the next one $\left(8^{\text {th }}\right)$ will take place in August 2015 in Colombia. A new International Society of Medicinal Mushrooms was registered in 2013 in Canada.

Approximately 400 clinical trial studies using MMs have been published on different illnesses. More than 50,000 scientific papers have been published and approximately 15,000 patents dedicated to studying different aspects of MMs have been received. From 2005, each year 250-350 patents have been registered for Ga. lucidum. ${ }^{[51}$ 
Taiwanese scientists received more than 100 patents on one species of genus Antrodia.

On the other hand, there are many unsolved, serious problems in the future of MM development, which in turn can also affect the continuation of MM science in the $21^{\text {st }}$ century. Below, the most critically important problems in the continuing development of MM science are listed.

\section{Taxonomy and nomenclature of MMs}

The father of biological nomenclature, Professor C. Linnaeus, wrote 300 years ago "Nomina si nescis, perit et cogito rerum" (If you don't know the species name, you don't understand the core).

Many species of MMs are critically misunderstood. Without the correct scientific name of the MM, future investigative studies will have no validity. Together with classical taxonomical methods, DNA bar coding may be useful and helpful for the correct identification of MM species, including the study of type material and standardized MM products. For example, there is now mounting evidence that shows most species previously reported as Lingzhi or Reishi (Ga. lucidum) in most pharmacological studies were mistakenly identified. Ga. lucidum presents a taxon-linneon or species-complex of which the future subdivision requires caution. ${ }^{[52]}$ Publications, patents, and products are also at risk. Over the years, at least 166 laccate Ganoderma species have been described worldwide. ${ }^{[53]} \mathrm{Ap}-$ proximately 100 Ganoderma species are known from China. ${ }^{[54]}$ It is not known what the taxonomic positions of so-called medicinal Blue Ling Zhi, or Red Ling Zhi, or White Ling Zhi are, for example.

Another important point to be noted is that Cao and his colleagues published a paper in which they claimed that the so-called $G a{ }^{[55]}$ lucidum in China is quite different from the Ga. lucidum found and described from Europe, and so they introduced a new species, Ganoderma lingzhi Sheng H. Wu, Y. Cao et Y. C. Dai, for Chinese mycobiota. This brought more problems and confusion. Who knows which Ganoderma species is medicinal? Which species of Lingzhi is the Chinese national mushroom Ga. lucidum or Ga. lingzhi? Nobody knows. Young and Feng published a special mini-review dedicated to this problem, called "What is the Chinese 'Lingzhi'?"[56] Molecular phylogenetic analyses based on the ITS (internal transcribed spacer) and 25S ribosomal DNA sequences indicated that most of the collections called "Ga. lucidum" in East Asia were not conspecific with Ga. lucidum found in Europe. ${ }^{[56]}$

Moreover, in 2012, in Nature Communications, a group of scientists published a paper dedicated to genome sequences of the model medicinal mushroom Ga. lucidum. ${ }^{[57]}$ Authors reported Ga. lucidum 43.3-Mb genome, encoding 16,113 predicted genes, obtained using next-generation sequencing and optical mapping approaches. However, this very important publication did not solve the problem of the Ga. lucidum species complex because authors studied one dik ariotic strain, CGMCC5.0026, belonging to the $\mathrm{Ga}$. lucidum Asian group from China, which was claimed as the new species to science, Ga. lingzhi.

Another example of mistaken identity was revealed for A. blazei, well known in literature as a MM. A. blazei is a North American endemic species described only from three localities and does not exist in culture; therefore, it cannot be listed as a MM. Two different opinions of A. blazei exist: A. blazei sensu Murrill, reported from three localities of the USA, and A. blazei sensu Heinem., reported from Brazil and cultivated in Japan. ${ }^{[42,43]}$ We studied the type material of A. blazei sensu Murrill, A. blazei sensu Heinem., Agaricus subrufescens from the New York Botanical Garden (NY), and other species of this group, as well as cultivated strains from different countries and the material from nature in Brazil. A. blazei sensu Murrill and A. blazei sensu Heinem. represent two different species. A. blazei sensu Murrill differs from A. blazei sensu Heinem. in size, shape of fruit bodies, pileal surface, type of pileal covering, presence of cheilocystidia, and spore size. It was determined that the widely cultivated culinary-medicinal mushroom known as A. blaze $i$ had nothing in common with the A. blazei described by Murrill from the USA, and therefore, a new species to science was described as A. brasiliensis. ${ }^{[42,43]}$ A. blaze $i$ is no longer known as a culinary-medicinal mushroom. Later, using morphologic data with molecular and biological data, the differences between A. blazei and A. brasiliensis were proven. ${ }^{[43]}$ Kerrigan $^{[58]}$ and our researchers ${ }^{[42,43,59]}$ published several articles in the last few years to clarify distinctions among A. brasiliensis, A. subrufescens, Agaricus fiardii, Agaricus praemagniceps, and A. blazei. These species are now classified with distinct morphologic, molecular, and biological characteristics and different geographic distribution. The misclassification of A. blazei caused many problems in MM science, but has since been corrected. I would like to bring to your attention the incorrect use of the name A. blazei for culinary-medicinal Royal Sun Agaricus or the Himematsutake mushroom. A. blazei is not a medicinal mushroom; this species does not exist in culture and is known only from three localities in the USA.

It is a pity that even today in many papers, including Chinese papers, scientists are using the wrong name, A. blazei, instead of A. brasiliensis for the medicinal species Royal Sun Agaricus.

\section{The study of culinary- MMs in pure culture}

More attention must be paid to the study of culinaryMMs in pure culture. The study of cultures is necessary to provide stability and continuity in scientific work. The 
teleomorph stage is the most essential criterion for the identification of cultures sometimes MMs do not produce fruit bodies in pure culture. Vegetative mycelia of MMs in pure culture have received little attention in mycological literature. Many species of MMs cannot be identified correctly without the study of vegetative mycelia. Vegetative mycelia of MM cultures are complexes of branched hyphae, which differ only within narrow limits of width, length, number of nuclei, thickness of cell wall, and branching. Gathering information on an increasing number of studies of vegetative mycelia of $\mathrm{MM}$ species provides new material for the study and comparison of morphologic characteristics of mycelia and for the estimation of their potential use for taxonomic purposes and purity control in biotechnological processes. ${ }^{[3,4,60]}$ Because there are no type strains of MMs, we need to choose the correctly identified type strains of many species of MMs. We need to organize a world culture collection of culinary- MMs, with depository activity following patent procedures according to the Budapest Treaty. This issue must be discussed with the World Federation of Culture Collections. ${ }^{[3,4]}$

\section{DS problems}

Recently, there has been growing popularity in developing mushroom biomass or different extracts as DSs or functional foods and novel prebiotics (non-digestive $\beta$-glucans). Significant questions arise with establishing DS and MM products, including their safety, standardization, regulation, efficacy, and mechanism of action.

Unfortunately, standardization around the world of DSs from MMs is still in its early stages, including an insufficient understanding of the bioactive effects of DS. We do not have internationally recognized standards and protocols for the production and testing of MM products. Only proper standards and protocols can guarantee product quality. Without consistency in the quality of MM products, commercially available MM preparations of mushrooms will be dramatically different and vary enormously in composition and affectivity. It is not known whether bioactive effects are caused by a single component or are the result of a synergistic impact of several ingredients. There is insufficient data to determine which components have better effects - those from mushroom fruiting bodies or from submerged mycelia powder versus extracts. Are simple dried fruiting bodies and mycelia powders as affective as hot water, alcoholic, or hydro-alcoholic extracts? Between crude extracts and isolated fractions, which one is more affective and has a higher safety profile (some companies are selling fractions like $G r$. frondosa Maitake D-fraction or GLPS fraction of Ga. lucidum)? The role of low-molecular-weight compounds for MM extracts is still unclear.

What is more affective - the combination of components containing biomass or extracts of 2-10 different species in one pill, or having one species in one pill? How can one assess the effectiveness of different mushroom products when blending in many species in one product ("shotgun" approach)? Since mushroom products can be cytokine stimulants, what is the age from which they can be safely administered to young children, since their immune systems are not yet mature?

Data regarding the dosage used are controversial. The suggested dosages are highly different due to various forms and formulations. Numerous clinical trials have established that six capsules (three capsules two times per day or two capsules three times per day), of 500-1000 mg each (biomass or extracts), is the accepted dosage of MM preparations. According to traditional Chinese medicine (TCM), the standard dose of the dried fruiting bodies of biomass of MMs per day in different forms (tablets, capsules, liquid extracts, etc.) must be equivalent to about 100-150 g of fresh mushroom material.

What dosages are safe and affective during pregnancy and nursing? The absence of sufficiently elaborated standards for the recommended use of MM DSs, including precise doses and duration of administration, needs very serious investigation. Some research shows that too high a dose could lead to immune suppression and too low a dose might not trigger an immune response.

Furthermore, major problems associated with mushroom-based DSs are due to their wide variability, the current lack of standards for production, and the lack of testing protocols necessary to guarantee product quality. The active ingredients of many present-day commercial mushroom products have not been indicated.

Insufficient results due to the lack of standards for production and product quality of commercially available mushroom preparation from the same species and strains of mushrooms are dramatically different in composition and affectivity. Adulteration of MM products with similar or spurious species (for instance, different Ganoderma species for Ga. lucidum; Stereum species for T. versicolor; different Cordyceps species and even different anamorphic species of Cordyceps for Ophiocordyceps sinensis) is very common.

There are difficulties in producing pure $\beta$-glucans for the market (90-95\% of $\beta$-glucan on the market is considered counterfeit and adulterated). Adulteration has led to a number of adverse effects resulting in nephropathy, acute hepatitis, coma, and fever. ${ }^{[3,4,61]}$

Still, we have not solved the problems concerning the safety of several well-known MM products. For example, on the basis of a study of a Phase I/II trial polysaccharide extract of Gr. frondosa in breast cancer patients, it was concluded that the Maitake mushroom produces more complex effects than presumed and may depress as well as enhance immune function. ${ }^{[62]}$ 
What is the role of fresh mushroom consumption? The consumption of fresh mushrooms has been found to increase, for example, anti- $\beta$-glucan antibodies in the serum of humans. The Ohno group from Japan also suggested that consumption of fresh mushrooms would provide better defense against pathogenic fungi. ${ }^{[63]}$

Information is still lacking on the use of MM DSs and their inter-crossing or interaction with many common drugs.

\section{MM natural products are an unclaimed source for drug discovery}

The development of real immunomodulating and anticancer drugs from MM polysaccharides (e.g. Lentinan, Schizophyllan, and Krestin) was hampered by the fact that high-molecular-weight compounds had been used. ${ }^{[23,24]}$ All MM drugs were developed from polysaccharides of high-molecular-weight, ranging from 100,000 to 0.5 million Da. These compounds cannot be synthesized; therefore, their production is restricted to extraction from fruit bodies, cultured mycelium, or cultured broth. Such an approach imposes high market prices. Today, science should concentrate on the beneficial medicinal effects of low-weight-molecular compounds produced by MMs (i.e. low-molecular-weight secondary metabolites targeting processes such as apoptosis, angiogenesis, metastasis, cell cycle regulation, and signal transduction cascades). ${ }^{[16]}$ Western pharmaceutical companies are more interested in relatively easily synthesized compounds that can be produced for markets.

Historically, the majority of new drugs have been generated from natural products (secondary metabolites). MMs are an unclaimed source for drug discovery. By 1990, about $80 \%$ of drugs were either natural products or analogs inspired by them. "Blockbuster drugs" like antibiotics (penicillin, tetracycline, and erythromycin), antiparasitics (avermectin), anti-malarials (quinine, artemisinin), lipid control agents (lovastatin and analogs), immunosuppressants for organ transplants (cyclosporin, rapamycins), and anticancer drugs (taxol, doxorubicin) revolutionized medicine. ${ }^{[64]}$ Many of the above-mentioned drugs were discovered from components found in fungi.

Modern pharmaceutical trends in preventing cancer include the development of new drugs with the following: (A) Growth factor inhibitors of cancer cells (drugs such as herceptin, erbitux, and terceva); they block a cancer cell's link to critical proteins that help it divide and grow; (B) hormone blockers (drugs such as tamoxifen) keep cells from dividing by binding to estrogen receptors, which are over-expressed in some tumor cells; (C) signal blockers working inside a cell; these drugs interrupt communication among enzymes that regulate growth and development; and (D) angiogenesis inhibitors (e.g. avastin was the first drug to inhibit the formation of new blood vessels around cancer cells, starving them of nourishment). ${ }^{[65]}$ About 860 cancer drugs are being tested on humans. This number is more than twice the number of experimental drugs for heart disease and stroke combined, nearly twice as many for AIDS and all other infectious diseases combined, and nearly twice as many for Alzheimer's and all other neurological diseases combined. ${ }^{[66]}$ Cancer drugs have been the biggest category of drugs in terms of sales worldwide since 2006 and in the US since 2008, according to market research by IMS Health. Today, drug companies see a future in treating cancer. The world's largest pharmaceutical company (Pfizer), for example, was focused on cardiovascular drugs, the cholesterol-lowering buster Lipitor, ${ }^{[67]}$ and the blood pressure reduction pill Norvasc. ${ }^{[6]}$ Recently, Pfizer hired about 1000 researchers for an all-out effort to develop drugs for cancer, a disease the company once largely ignored. Pfizer has now scaled back on cardiovascular research and has made cancer drugs one of its six focus areas. About $20 \%$ of Pfizer's more than $\$ 7$ billion budget for research and development is on cancer research, and 22 of the roughly 100 drugs being tested are anticancer drugs. ${ }^{[66]}$

Progress in research of MMs must include genomics, proteomics, metabolomics, and systems pharmacology. Studying the molecular mechanisms to determine the medicinal effects of MMs should be the focus of new investigations using modern methods in the above approaches.

Another important source for substances of therapeutic interest can be found in the pool of secondary metabolites produced by MMs. These substances can be classified according to five main metabolic sources: ${ }^{[16]}$ Amino acid-derived pathways; the shikimic acid pathway for the biosynthesis of aromatic amino acids; acetate-malonate pathway from acetyl coenzyme A; the mevalonic acid pathway from acetyl coenzyme A, which functions in the primary metabolism for the synthesis of sterols; and polysaccharides and peptidopolysaccharides. The polyketide and the mevalonic acid pathways are most often involved, and they produce a greater variety of compounds than the other pathways.

Every effort should be made to find new sources for anticancer drugs using low-molecular-weight secondary metabolites from MMs that can inhibit or trigger specific responses, i.e. activating or inhibiting $\mathrm{NF}-\kappa \mathrm{B}$, inhibiting proteins, especially tyrosine kinases, aromatase and sulfatase, matrix metalloproteinases, cyclooxygenases, DNA topoisomerases, and DNA polymerase, anti-angiogenic substances, etc. ${ }^{[16,45-50]}$

The fungal low-molecular-weight compounds directly influencing NF- $\kappa b$ inhibitory effects are: CAPE, cordycepin, panepoxydone, and cycloepoxydon. Low-molecular-weight CAPE produced, for example, by $P$. linteus and $M$. oreades shows specific cytotoxicity against tumor cells and NF-Kb inhibitor activity, and can be a candidate for antitumor drugs, especially against breast cancer. ${ }^{[46,50]}$

Pharmaceutical companies involved in drug discovery 
need new sources of natural products. MMs are the best unclaimed gifts of nature that in a short amount of time can be used in the production of new pharmaceuticals. Here the author presents the Drug Discovery Pathway, which was especially prepared for the development of MM pharmaceuticals. This pathway includes nine steps:

1. Mushroom cultivation and biomass production

2. Biomass extraction

3. Screening of mushroom extracts

4. Effect of selected extracts on a target of interest

5. Chemical fractionation of selected extracts

6. Elucidation of active fractions (compounds), mechanism of action, and potency

7. Effect on animal models

8. Preclinical drug development

9. Clinical drug development. ${ }^{[3]}$

\section{Unsolved problems in the study of structural characteristics, isolation process, receptor-mediated mechanism, and antitumor activity of MM $\beta$-glucans}

The success of application of $\beta$-glucans and other mushroom carbohydrate polymers requires active research in addressing the structure-activity relationship of mushroom carbohydrate polymers, especially in terms of molecular conformation and receptor-mediated mechanisms. ${ }^{[25,68]}$ Clarification of water solubility, size of molecules and molecular weight, structure, and molecular mechanisms of $\beta$-glucan action takes into consideration that not all $\beta$-glucans contained in a MM exhibit pharmaceutical activity. ${ }^{[22,68,69]}$

The role of molecular weight in the pharmaceutical activity of $\beta$-glucans is still not known. Studies dedicated to comparing the affectivity of high-molecular-weight B-glucans versus low-molecular-weight B-glucans are still being investigated. Scleroglucan high-molecular-weight preparations are most effective. ${ }^{[69]}$ But, for example, only low-molecular-weight Lentinan has higher antitumor activity. ${ }^{[21,22]}$ The different reactivity of $\beta$-glucans in each individual must be taken into account (anti- $\beta$-glucan titer and increments of the titer by $\beta$-glucan administration are different; reactivity of peripheral blood leukocytes to $\beta$-glucan is significantly different in each individual; reactivity to $\beta$-glucans is, for example, significantly different in various strains of mice). ${ }^{[22,68,69]}$

Solubility in water is one of the important characteristics of $\beta$-glucans. It is still unknown what are the major factors affecting the solubility and pharmaceutical activity of $\beta$-glucans; molecular weight, length of side chain, number of side chains on the main chain, ratios of $(1,4),(1,6)$ and $(1,3)$ linkages, and ionization by acid must be considered. ${ }^{[3,22,68]}$ Soluble $\beta$-glucans appear to be stronger immunostimulators than insoluble $\beta$-glucans. The reasons for this are not totally clear. The exact mechanism of intestinal absorption of orally administered $\beta$-glucan remains unknown (nonspecific intestinal absorption, passage of $\beta$-glucans through the gap junction in the intestinal epithelial membrane, absorption through intestinal $\mathrm{M}$ cells, absorption after binding with Toll-like receptor proteins on the intestinal lumen, and dendritic cell probing). ${ }^{[70,71]}$ It is possible that orally administered insoluble $\beta$-glucans are subsequently degraded into smaller bioactive oligomers after ingestion. ${ }^{[72]}$

The differences between plant $\beta$-glucans, ${ }^{[73,74]}$ yeasts $\beta$-glucans, ${ }^{[75]}$ and $\beta$-glucans from MMs ${ }^{[3,22,25,68,69]}$ must be clarified. What is the difference in structure, solubility, and biological activity? For example, the structure of cereal $\beta$-glucan is essentially $\beta-1,3$ and $\beta-1,4$ linkages, not $\beta-1,6$ linkages. In addition, plant $\beta$-glucans are linear, not branched. Usually, the molecular weights of plant $\beta$-glucans are smaller than those of MM $\beta$-glucans. Biological activity has not been fully examined in the case of plant $\beta$-glucans. Usually, yeast $\beta$-glucans are only partly water-soluble and many MM $\beta$-glucans are water insoluble. Why do they have different biological activities? What are the key advantages of MM $\beta$-glucans compared to cereal $\beta$-glucans or yeast $\beta$-glucans?

We know a lot about the function of receptor dectin-1 (dendritic-cell-associated C-type lectin-1) of $\beta$-glucans. ${ }^{[25,76,77]} \beta$-glucans have antifungal activity that is similar to their anticancer activities and is mediated by binding to dectin- 1 . However, it is still not known how $\beta$-glucans bind to receptor dectin-1. We know a lot about the function of receptor dectin- 1 of $\beta$-glucans, but the function of receptor dectin-2 is still unclear. ${ }^{[25,78]}$

Why do $\beta$-glucans have the triple-helix conformation, and does the triple-helix structure have an advantage for MMs that have a single strand ? $^{[3,22,68,69]}$ Unfortunately, we do not understand what structural features are best for inducing specific activities and, even more importantly, what does the presence of hydrophilic groups on the outside surface of the helix mean. We can see contradictory data in literature on the biological activity of triple-helix and single-helix structures of the same $\beta$-glucan - for example, Schizophyllan. ${ }^{[22,68,69]}$ We still do not know which has stronger biological activity - the closed triple helix or a partially opened triple helix. ${ }^{[28,79]}$ Some species form triple helix aqueous solutions. However, in the presence of alkaline pH or DMSO (dimethyl sulfoxide) the triple helix converts rapidly to a single-stranded helix, which gradually reforms a triple helix at neutral $\mathrm{pH}$. However, the form of the helix associated with more effective immunomodulatory and antitumor activities is unknown. ${ }^{[25]}$

\section{Conclusions}

1. The role of polysaccharide-protein or polysaccharide-peptide complexes in the pharmacological activity of MM needs further investigation

2. More studies are needed to demonstrate which 
mushroom extracts or compounds are most affective for specific ailments against viral infections, bacterial infections, metabolic syndromes, cancer, cholesterol, etc

3. The development of new methods and processes in the study of MMs must be a priority

For example, a new method was developed in 2009 for nanoparticle extraction of water-soluble $\beta$-glucans from MMs by the Park group ${ }^{[80]}$ from South Korea. A novel process for nanoparticle extraction of Sparan, the $\beta$-d-glucan from Sparassis crispa, and Phellian, the $\beta$-d-glucan from $P h$. linteus, was investigated using insoluble tungsten carbide as a model for nanoknife technology. This was the first report showing that the nanoknife method results in high yields of Sparan (70.2\%) and Phellin (65.2\%) with an average particle size of 150 and $390 \mathrm{~nm}$, respectively. The Park group proposed the nanoknife method could be used in producing $\beta$-glucans for food, cosmetics, and pharmaceutical industries. German scientists ${ }^{[81]}$ developed a new colorimetric method to quantify $\beta-1,3-1,6$ glucans in comparison with total $\beta-1,3$ glucans and a method to quantify chitin in edible mushrooms.

4. The discovery of the new species of mushrooms with pharmaceutical activity must be our priority. Only the last few years, scientists have found new mushroom species, with pharmacological activities, for example, M. oreades, Trametes ochracea, Xylaria nigrepes, Pseudotrameres (=Daedalea) gibbosa, Geastrum saccata, Cyathus striatus, and Cyathus olla $^{[46,82,83]}$

5. MM use for humans has made very little progress until now. High-quality long-term double-blind placebo-controlled clinical studies of MMs, including well-sized population studies to yield statistical power showing efficacy and safety, are definitely needed. Aims of clinical trials during Phase I, II, III, and IV must be to obtain sufficient data on the efficacy and safety of MM drugs and preparations

6. More attention must be paid to do research on farm animals and MMs. On the one hand, there are research areas that could potentially be advanced by using farm animals as biomedical models including obesity, diabetes, aging, cardiovascular diseases, infectious diseases, neurobiology, cancer, nutrition, immunology, ophthalmology, and reproduction. On the other hand, we can revolutionize farm animal research, which is now in crisis, ${ }^{[84]}$ by proposing new types of food and DS, antibiotic replacement, and antiviral agents for farm animals

7. More attention must be paid to do research on MMs as agricultural insecticides and anti-plant virus compositions

8. Protecting the intellectual properties (IPs) of MM genetic resources for invention and innovation is a problem that needs more attention. Mushroom genetic resources are currently being utilized and exploited by the pharmaceutical, cosmetic, agricultural, food, enzyme, chemical, and waste-treatment industries. Nevertheless, the role of IPs' advantages in today's knowledge-driven enterprises is frequently overlooked, despite their potential as sources of monetary value and financial gain. IPs are often under-managed or under-leveraged. The challenge is how to create, protect, and extract value from IP assets for invention and innovation ${ }^{[3,85]}$

9. We must continue to educate the society and consumers on MM science

It is our responsibility as scientists to do much more in educating the public at large on the health benefits of MMs. Interest in and advancements made in current research are not always visible or available to the public. It is hard to believe that in 2014, many people all over the world are completely unaware of the health benefits of MMs.

10. To our advantage, we should bridge the gap between Western and Eastern medicine.

Western and Eastern medicines have adopted different regulatory systems for herbal and mushroom preparations. Most Western countries follow the rules of the WHO and DSHEA (Dietary Supplement Health and Education Act) in which plant or MM extracts are defined as DSs and clinical studies are not required before DSs are introduced on the market. China and several other Asian countries define many of the same herbs and some MMs as drugs, and therefore, clinical studies are needed. Western medicine has made little use of MM products partly due to their complex structure and lack of acceptable pharmacological purity. Our target for the future should be to adopt those regulations, standards, and practices from Western and Eastern medicine that have proven to be the most valuable in the quest for health benefits in the $21^{\text {st }}$ century. ${ }^{[3,4]}$

\section{REFERENCES}

1. Reshetnikov SV, Wasser SP, Tan KK. Higher Basidiomycota as source of antitumor and immunostimulating polysaccharides. Int J Med Mushrooms 2001;3:361-94.

2. Van Griensven LJ. Culinary-medicinal mushrooms: Must action be taken. Int J Med Mushrooms 2009;11:281-6.

3. Wasser SP. Medicinal mushroom science: History, current status, future trends, and unsolved problems. Int J Med Mushrooms 2010;12:1-16.

4. Chang ST, Wasser SP. The role of culinary-medicinal mushrooms on human welfare with a pyramid model for human health. Int J Med Mushrooms 2012;1:95-134.

5. Pöder R. The Ice man's fungi: Facts and mysteries. Int J Med Mushrooms 2005;7:357-9.

6. Wasson RG. Soma. Divine mushroom of immortality. NY, USA: 
Harcourt Brace Jovanovich, Inc.; 1968.

7. Hyde KD, Bahkali AH, Moslem MA. Fungi- an unusual source for cosmetics. Fungal Divers 2010;43:1-9.

8. Lindequist $U$. The merit of medicinal mushrooms from a pharmaceutical point of view. Int J Med Mushrooms 2013;15:517-23.

9. Hawksworth DL. Mushrooms: The extent of the unexplored potential. Int J Med Mushrooms 2001;3:333-40.

10. Hawksworth DL. Global species number of fungi: Are tropical studies and molecular approaches contributing to a more robust estimate? Biodivers Conserv 2012;21:2425-33.

11. Kirk PM, Cannon PF, David JC, Stalpers JA. Ainsworth and Brisby's Dictionary of the Fungi. $10^{\text {th }}$ ed. Wallingford: CAB International; 2008.

12. Blackwell M. The fungi: $1,2,3$...5.1 million species? Am J Bot 2011;98:426-38.

13. Bass D, Richards TA. Three reasons to re-evaluate fungal diversity 'on Earth and in the ocean'. Fungal Biol Rev 2011;25:159-64.

14. Hibbett DS, Taylor JW. Fungal systematics: Is a new age of enlightenment at hand? Nat Rev Microbiol 2013;11:129-33.

15. Anke T. Basidiomycetes: A source for new bioactive secondary metabolites. Prog Ind Microbiol 1989;27:51-66.

16. Zaidman BZ, Yassin M, Mahajna J, Wasser SP. Medicinal mushroom modulators of molecular targets as cancer therapeutics. Appl Microbiol Biotechnol 2005;67:453-68.

17. De Silva DD, Rapior S, Sudarman E, Stadler M, Xu J, Alias SA, et al. Bioactive metabolites from macrofungi: Ethnopharmacology, biological activities and chemistry. Fungal Divers 2013;62:1-40.

18. Dai YC, Yang ZL, Ui BK, Yu CH, Zhou LW. Species diversity and utilization of medicinal mushrooms and fungi in China (review). Int J Med Mushrooms 2009;11:287-302.

19. Lo HC, Wasser SP. Medicinal mushrooms for glycemic control in diabetes mellitus: History, current status, future perspectives, and unsolved problems (review). Int J Med Mushrooms 2011;13:401-26.

20. Didukh MY, Wasser SP, Nevo E. Medicinal value of species of the family Agaricaceae Cohn (higher Basidiomycetes) current stage of knowledge and future perspectives. Int J Med Mushrooms 2003;5:133-52.

21. Chihara G, Hamuia J, Maeda YY, Arai Y, Fukuoka F. Fractionation and purification of the polysaccharides with marked antitumor activity, especially lentinan, from Lentinus edodes (Berk.) Sing. (an edible mushroom). Cancer Res 1970;30:2776-81.

22. Zhang M, Cui SW, Cheung PC, Wang Q. Antitumor polysaccharides from mushrooms: A review on their isolation process, structural characteristics and antitumor activity. Trends Food Sci Technol 2007;18:4-19.

23. Zhang Y, Li S, Wang X, Zhang L, Cheung PC. Advances in lentinan: Isolation, structure, chain conformation and bioactivities. Food Hydrocoll 2011;25:1996-2006.

24. Zhang Y, Kong H, Fang Y, Nishinan K, Phillips GO. Schizophyllan: A review on its structure, properties, bioactivities and recent development. Bioact Carbohydr Diet Fiber 2013;1:53-71.

25. Lee DH, Kim HW. Innate immunity induced by fungal b-glucans via dectin-1 signaling pathway. Int J Med Mushrooms 2014;16:1-16.
26. Barillot E, Calzone L, Hupe Ph, Vert JPh, Zinovyev A. Computation system biology of cancer. USA: CRC Press, Taylor and Francis Group. A Chapman and Hall Book; 2013.

27. Stewart BW, Wild ChP, editors. World Cancer Report 2014. Lyon, France: International Agency for Research on Cancer; 2014.

28. Mizuno T. The extraction and development of antitumor-active polysaccharides from medicinal mushrooms in Japan (Review). Int J Med Mushrooms 1999;1:9-29.

29. Hobbs C. Medicinal value of Lentinus edodes (Berk.) Sing. (Agaricomycetideae). A literature review. Int J Med Mushrooms 2000;2:287-302.

30. Zhuang C, Wasser SP. Medicinal value of culinary-medicinal Maitake mushroom Grifola frondosa (Dicks.:Fr.) S.F.Gray (Aphyllophoromycetideae). Review. Int J Med Mushrooms 2004;6:287-313

31. Boh B, Berivic M. Grifola frondosa (Diks.:Fr.) S.F. Gray (Maitake mushroom): Medicinal properties, active compounds, and biotechnological cultivation. Int J Med Mushrooms 2007;9:89-108

32. Hobbs C. The chemistry, nutritional value, immunopharmacology, and safety of the traditional food of medicinal split-gill fungus Schizophyllum commune Fr.:Fr. (Aphyllophoromycetideae). A literature review. Int J Med Mushrooms 2005;7:127-40.

33. Lin ZB. Lingzhi. From mystery to science. Beijing, China: Peking University Press; 2009.

34. Mahajna J, Dotan N, Zaidman BZ, Petrova RD, Wasser SP. Pharmacological values of medicinal mushrooms for prostate cancer therapy: The case of Ganoderma lucidum. Nutr Cancer 2010;61:16-26.

35. Hobbs C. Medicinal value of Turkey Tail fungus Trametes versicolor (L.:Fr.) Pilát (Aphyllophoromycetideae). Int J Med Mushrooms 2004;6:195-218.

36. Mizuno T, Zhuang C, Abe K, Okamoto H, Kiho T, Ukai S, et al. Antitumor and hypoglycemic activities of polysaccharides from the sclerotia and mycelia of Inonotus obliquus (Pers.:Fr.) Pil. (Aphyllophoromycetideae). Int J Med Mushrooms 1999;1:301-16.

37. Balandykin ME, Zmitrovich IV. Review on Inonotus obliquus (Basidiomycota). Realm on medicinal applications and approaches on resources estimation. Int J Med Mushrooms 2014;16 (In Press).

38. Hsieh PW, Wu JB, Wu YC. Chemistry and biology of Phellinus linteus. BioMed 2013;3:106-13.

39. Maruyama $\mathrm{H}$, Ikekawa $\mathrm{T}$. Immunomodulation and antitumor activity of a mushroom product, proflamin, isolated from Flammulina velutipes (W.Curt.:Fr.) Singer (Agaricomycetideae). Int J Med Mushrooms 2007;9:109-22.

40. Matsuzawa T. Studies on antioxidant effects of culinary-medicinal bunashimeji mushroom Hypsizygus marmoreus (Peck) Bigel. (Agaricomycetidae). Int J Med Mushrooms 2006;8:245-50.

41. Holliday H, Cleaver M. Medicinal value of the caterpillar fungi species of the genus Cordyceps (Fr.) Link (Ascomycetes). A review. Int J Med Mushrooms 2008;10:209-18.

42. Wasser SP, Didukh MY, Amazonas MA, Nevo E, Stamets P, Eira AF. Is widely cultivated culinary-medicinal Royal Sun Agaricus (the Himematsutake mushroom) indeed Agaricus blazei Murrill? Int J Med Mushrooms 2002;4:267-90.

43. Wasser SP, Didukh MY, Amazonas MA, Nevo E, Stamets P, Eira AF. Is a widely cultivated culinary-medicinal Royal Sun Agaricus (Champignon do Brazil, or the Himematsutake mushroom)

Biomed J Vol. 37 No. 6

November - December 2014 
Agaricus brasiliensis S. Wasser et al. indeed a synonym of A. subrufescens Peck? Int J Med Mushrooms 2005;7:507-11.

44. Lachter J, Yampolsky Y, Gafni-Schieber R, Wasser SP. Yellow Brain Culinary-Medicinal Mushroom, Tremella mesenterica Ritz.:Fr. (Higher Basidiomycetes), is subjectively but not objectively effective for eradication of Helicobacter pylori; a prospective controlled trial. Int J Med Mushrooms 2012;14:55-63.

45. Yassin M, Wasser SP, Mahajna J, Ruimi N, Denchev CM, Sussan S, et al. Substances from the medicinal mushroom Daedalea gibbosa inhibit kinase activity of native and T315I mutated Bcr-Abl. Int J Oncol 2008;32:1197-204.

46. Petrova RD, Mahajna J, Wasser SP, Ruimi N, Denchev CM, Sussan S, et al. Marasmius oreades substances block NF-kappaB activity through interference with IKK activation pathway. Mol Biol Rep 2009;36:737-44

47. Rouhana-Toubi A, Wasser SP, Fares F. Ethyl acetate extracts of submerged cultured mycelium of higher Basidiomycetes mushrooms inhibit human ovarian cancer cell growth. Int J Med Mushrooms 2009;11:29-37.

48. Dotan N, Wasser SP, Mahajna J. The culinary-medicinal mushroom Coprinus comatus as a natural antiandrogenic modulator. Integr Cancer Ther 2011;10:148-59.

49. Ruimi N, Petrova RD, Agbaria R, Sussan S, Wasser SP, Reznick AZ, et al. Inhibition of TNFo-induced iNOS expression in HSV-tk transduced 9L glioblastoma cell lines by Marasmius oreades substances through NF-kB- and MAPK-dependent mechanisms. Mol Biol Rep 2010;37:3801-12.

50. Ruimi N, Rwashdeh H, Wasser SP, Konkimalia B, Efferth T, Borgatti M, et al. Daedalea gibbosa substances inhibit LPS-induced expression of iNOS by suppression of NF- $\mathrm{KB}$ and MAPK activities in RAW 264.7 macrophage cells. Int J Mol Med 2010;25:421-32.

51. Boh B. Ganoderma lucidum: A potential for biotechnological production of anti-cancer and immunomodulatory drugs. Recent Pat Anticancer Drug Discov 2013;8:255-87.

52. Wasser SP, Zmitrovich IV, Didukh MY, Spirin WA, Malysheva VF. Morphologigal traits of Ganoderma lucidum complex highlighting G. tsugae var. jannieae: The current generalization. Ruggell, Liechtenstein: A.R.A. Gantner Verlag K.-G.; 2006.

53. Moncalvo JM, Rivarden L. A nomenclatural study of the Ganodermataeae. Fungiflora A/S Oslo:; Norway, 1997.

54. Zhao JD. The Ganodermataceae of China. Bibliotheca Mycologica 132. Gebrüder Borntraeger, Berlin, Stuttgart: 1989.

55. Cao Y, Wu SH, Dai YC. Species clarification of the prize medicinal Ganoderma mushroom "Lingzhi”. Fungal Divers 2012;56:49-62.

56. Yang ZL, Feng B. What is the Chinese "Lingzhi"? - a taxonomic mini-review. Mycology 2013;4:1-4.

57. Chen Sh, Xu J, Liu C, Zhu Y, Nelson DR, Zhou S, et al. Genome sequence of the model medicinal mushroom Ganoderma lucidum. Nature communications 2012;3:913.

58. Kerrigan RW. Agaricus subrufescens, a cultivated edible and medicinal mushroom, and its synonyms. Mycologia 2005;97:12-24.

59. Wasser SP. Molecular identification of species of the genus Agaricus. Why should we look at morpholgy? Int J Med Mushrooms 2007;9:85-8.

60. Buchalo AS, Mykchaylova O, Lomberg M, Wasser SP. Microstructures of vegetative mycelium of macromycetes in pure culture. Kiev, Ukraine; AlterPress; 2009.
61. McKenna DJ, Jones K, Hughes K, Humphrey S. Botanical medicines The desk reference for major herbal supplements. $2^{\text {nd }}$ ed. NY, London, Oxford: The Haworth Herbal Press; 2002.

62. Deng G, Lim H, Seidman A, Fornier M, D'Andrea G, Wesa K, et al. A phase I/II trial of a polysaccharide extract from Grifola frondosa (Maitake mushroom) in breast cancer patients: Immunological effects. J Cancer Res Clin Oncol 2009;135:1215-21.

63. Ishibashi KI, Dogasaki C, Iriki T, Motoi M, Kurone YI, Miura NN, et al. Anti- $\beta$-glucan antibody in bovine sera. Int J Med Mushrooms $2005 ; 7: 513$

64. Li JW, Vederas JC. Drug discovery and natural products: End of an era or an endless frontier. Science 2009;325:161-5.

65. Ammerpohl O, Tiwari S, Kalthoff $\mathrm{H}$. Target gene discovery for novel therapeutic agents in cancer treatment. Methods Mol Biol 2010;576:427-45.

66. Pollack A. Drug firms see fortune in treating cancer. Int Herald Tribune 2009; 15-16

67. Endo A. The origin of the statins. 2004. Atheroscler Suppl 2004:5:125-30

68. Chen J, Seviour R. Medicinal importance of fungal $\beta-(1-3)$, (1-6)-glucans. Mycol Res 2007;111:635-52.

69. Ohno N. Structural diversity and physiological functions of $\beta$-glucans. Int J Med Mushrooms 2005;7:167-73.

70. Miller H, Zhang J, Kuo Lee R, Patel GB, Chen W. Intestinal M cells: The fallible sentinels? World J Gastroenterol 2007;14:1477-86.

71. Pamer EG. Immune responses to commensal and environmental microbes. Nat Immunol 2007;8:1173-8

72. Lehmann J, Kunze R. Water-soluble low molecular weight beta-glucans for modulating immunological responses in mammalian system. US Patent 2000; 6143883.

73. Tada R, Adachi Y, Ishibashi KI, Tsubaki K, Ohno N. Binding capacity of a barley beta-D-glucan to the beta-glucan recognition molecule Dectin-1. J Agric Food Chem 208;56:1442-50.

74. Tiwari U, Cummins E. Factors influencing beta-glucan levels and molecular weight in cereal-based products. Cereal Chem 2009;86:290-301.

75. Liu JJ, Gunn L, Hansen R, Yan J. Combined yeast-derived beta glucan with anti-tumor monoclonal antibody for cancer immunotherapy. Exper Mol Pathol 2009;86:208-14

76. Taylor PR, Tsoni SV, Willment JA, Dennehy KM, Rosas M, Findon H, et al. Dectin-1 is required for beta-glucan recognition and control of fungal infection. Nat Immunol 2007;8:31-8.

77. Harada T, Ohno N. Dectin-1 and GM-CSF on immunomodulating activities of fungal 6-branched 1,3-ß-glucans. Int J Med Mushrooms 2008; $10: 101-14$

78. Graham LM, Brown GD. The Dectin-2 family of C-type lectins in immunity and homeostasis. Cytokine 2009;48:148-55.

79. Falch BH, Espevik T, Ryan L, Stokke BT. The cytokine stimulating activity of (1-3)-beta-D-glucans is dependent on the triple helix conformation. Carbohydr Res 2000;329:587-96.

80. Park HG, Shim YY, Choi SO, Park WM. New method development for nanoparticle extraction of water-soluble $\beta$-(1-3)-D-glucan from edible mushrooms, Sparassis crispa and Phellinus linteus. J Agric Food Chem 2009;57:2147-54 
81. Nitschke J, Modick H, Busch E, von Rekowski RW, Albenbach HJ, Mölleken H. A new colorimetric method to quantify b-1,3-1,6-glucans in comparison with total $\beta$-1,3-glucans in edible mushrooms. Food Chem 2011;127:791-6.

82. Khamaisie H, Sussan S, Tal M, Najajren Y, Rurhardt M, Mahajna J. Oleic acid is the active component in the mushroom Daedalea gibbosa inhibiting Bcr-Abl kinase autophosphorylation activity. Anticancer Res 2011;31:177-4.

83. Sharvit LE, Wasser SP, Fares F. The effect of culture liquid ethyl acetate mycelium extracts of medicinal mushrooms on the viability of human pancreatic cancer cells. Int J Med Mushrooms 2012;14:169-80

84. Roberts RM, Smith GW, Bazer FW, Cibelli J, Seidel GE Jr, Bauman DE, et al. Research priorities. Farm animal research in crisis. Science 2009;324:468-9.

85. Jong SC. Protecting intellectual property assest of mushroom genetic resources for invention and innovation. Int J Med Mushrooms 2005;7:348-9. 